\title{
NEW ALGORITHMS FOR DISCRETE-TIME OPTIMAL CONTROL PROBLEMS
}

\author{
NIKOLA B. NEDELJKOVIĆ
}

(Received 8 July 1983; revised 12 November 1983)

\begin{abstract}
The paper presents new demonstrably convergent first-order iterative algorithms for unconstrained discrete-time optimal control problems. The algorithms, which solve the linear-quadratic problem in one iterative step, are particularly suited for solving nonlinear problems with linear constraints via penalty function methods. The proofs of the reduction of cost at each iteration and convergence of the algorithms are provided.
\end{abstract}

\section{Introduction}

The purpose of this paper is to provide a new computational technique for solving discrete-time optimal control (or multi-stage decision) problems. Discrete-time systems are described by difference equations and involve choices or decisions at each of a finite set of times or stages. The optimal multi-stage decision problem is then to minimize the cost associated with each sequence of decisions.

Many economic and engineering problems in all sectors of business and industry can be viewed as multi-stage decision processes. Examples of this type of problem, where a finite change in control causes a finite change in the state of the system, include ecological systems, inventory control, resource allocation, production scheduling and the control of a system of water reservoirs.

\footnotetext{
${ }^{1}$ School of Mathematical and Physical Sciences, Murdoch University, Murdoch 6150, Western Australia.

(c) Copyright Australian Mathematical Society 1984, Serial-fee code 0334-2700/84
} 
As differential equations are harder to solve than difference equations, discrete approximations are sometimes used for solving continuous-time problems. Since both continuous and discrete-time controls can be considered as points in appropriate spaces, in view of the fact that the theory of functional analysis reflects to a degree our abstractions of intuitive geometric properties, it is not surprising that there are great similarities in the analyses for continuous and discrete-time systems. In general, formulas for the discrete-time case turn out to be somewhat more complex than their continuous-time counterparts, but are easier to justify as problems regarding the existence of solutions of differential equations do not arise.

Although difference equations are easier to solve than the differential equations, optimization of discrete-time systems is still a formidable task when the number of state and control variables and the number of stages and constraints is large. In [8], for instance, the Tennessee Valley Authority, which manages a system of 40 water reservoirs, reported that the maximum size of the severely constrained problem of controlling a system of water reservoirs solved with the existing numerical methods involved a system of 6 reservoirs. A general analytic solution of the discrete-time problem does not exist and recourse must be made to numerical methods. Dynamic programming, although impressive in comparison with direct enumeration, is effective only when the number of variables is small. Consequently, many iterative methods have been developed. Dyer and McReynolds [1] devised a second order method known as the successive sweep (SV) algorithm. Jacobson and Mayne [4] invented the differential dynamic programming (DDP) and Gershwin and Jacobson [2] analysed DDP for constrained problems and applied it to optimal orbit transfer. Since the SV algorithm [1] and the second order DDP algorithms in [4] and [2] are generally not convergent as they require the inversion of matrices which may be singular, Ohno [7] modified the second order discrete-time algorithm for problems with and without constraints and proved its local convergence (when the starting point is sufficiently close to the optimum). Recently, Wong and Teo [10] devised a computational method for optimal control problems with discrete delays and bounded control region.

The general characteristic of the existing first order methods, in comparison with second order methods, is that they require a large number of iterations. However, the second order methods require the computation of second order derivatives which is time consuming and necessitates a large computer memory; in fact, the computational effort per iterative step and memory requirements increase as a cubic function of the number of state and control variables. It is clear that a fast first order method would substantially increase our ability to 
solve large problems. One such technique is the algorithm in [9] developed by Teo, Wong and Clements for solving time-lag optimal control problems with control and terminal constraints. Control parametrization technique is for continuous time optimal control problems. However, it can also be applied to discrete time optimal control problems.

The aim of this paper is to present a fast first-order method for discrete-time systems. A number of rapidly convergent algorithms, known as LQRE, for continuous-time optimal control problems, was presented in [6]. Here we show that it is possible to develop discrete-time versions of the continuous-time LQRE algorithms. As the continuous-time algorithm in [6] is a first order method with the speed of convergence comparable to that of second order methods, its discrete-time analog (which is a globally convergent first order algorithm and converges on the linear-quadratic problem in one step) should be seen as a step in that direction.

The organization of the paper is as follows. Section 2 contains the definition of the problem. A statement of the proposed algorithm is given in Section 3. Section 4 is devoted to the proof of reduction in the value of the cost functional, whilst global convergence of the algorithm to a point satisfying a first order necessary condition for optimality is proved in Sections 5 and 6. Finally in Section 7 we show how the discrete-time LQRE algorithm in conjunction with the penalty function method can be applied to constrained problems in general and to problems with linear constraints in particular.

\section{Definitions and assumptions}

Let $N \geqslant 1$ be a fixed integer, let $U$ denote the space of admissible controls defined as the set of all ordered $N$-tuples $u \triangleq\left(u_{0}, u_{1}, \ldots, u_{N-1}\right), u_{k} \in R^{m}$, $k=0,1, \ldots, N-1$, and let $x \triangleq\left(x_{0}, x_{1}, \ldots, x_{N}\right), x_{k} \in R^{n}, k=0,1, \ldots, N$, denote the state of a system governed by a first order ordinary difference equation

$$
\begin{aligned}
x_{k+1} & =f\left(x_{k}, u_{k}, k\right), \quad k=0,1, \ldots, N-1, \\
x_{0} & =\chi_{0}, \quad \chi_{0} \text { specified. }
\end{aligned}
$$

The problem to be solved is the following.

Minimize the cost functional $J: U \rightarrow R$ defined by

$$
J(u)=\sum_{k=0}^{N-1} L\left(x_{k}, u_{k}, k\right)+F\left(x_{N}\right)
$$

subject to the system equation (2.1). 
Note that $u_{k}$ and $x_{k}$ are $m$ and $n$ dimensional vectors.

In the sequel $f\left(x_{k}, u_{k}, k\right)$ and $L\left(x_{k}, u_{k}, k\right)$ will also be denoted by $f_{k}\left(x_{k}, u_{k}\right)$ and $L_{k}\left(x_{k}, u_{k}\right)$, respectively. Since one can consider the above problem as a multi-stage process, it is customary to refer to $u_{k}$ and $x_{k}$ as the control and state of the system at stage $k$. We assume that $F: R^{n} \rightarrow R$ is continuously differentiable, that $f: R^{n} \times R^{m} \times \mathbf{N} \rightarrow R^{n}$ and $L: R^{n} \times R^{m} \times \mathbf{N} \rightarrow R$ are continuously differentiable in the first two arguments and that the solution to the discrete-time optimal control problem defined by (2.1) and (2.2) exists.

For the purpose of the analysis in this paper, it is convenient to write equations (2.1) and (2.2) in a slightly different form. Let $Q_{N}: R^{n} \rightarrow R^{n \times n}$ be continuously differentiable and let $A: R^{n} \times R^{m} \times \mathbf{N} \rightarrow R^{n \times n}, B: R^{n} \times R^{m} \times \mathbf{N} \rightarrow R^{n \times m}, Q$ : $R^{n} \times R^{m} \times \mathbf{N} \rightarrow R^{n \times n}, P: R^{n} \times R^{m} \times \mathbf{N} \rightarrow R^{m \times m}$ and $R: R^{n} \times R^{m} \times \mathbf{N} \rightarrow$ $R^{m \times m}$ be continuously differentiable in the first two arguments. In addition, let $Q(\chi, \omega, k), Q_{N}(\chi) \geqslant 0$ (positive semi-definite) and $R(\chi, \omega, k)>0$ (positive definite) for all $\chi \in R^{n}, \omega \in R^{m}$ and $k \in I_{N-1} \triangleq\{0,1, \ldots, N-1\}$. Without any loss of generality $Q(\chi, \omega, k), Q_{N}(\chi)$ and $R(\chi, \omega, k)$ can be considered as being symmetric. Then (2.1) and (2.2) can be rewritten as

$$
\begin{aligned}
x_{k+1} & =A\left(x_{k}, u_{k}, k\right) x_{k}+B\left(x_{k}, u_{k}, k\right) u_{k}+g\left(x_{k}, u_{k}, k\right), \quad k \in I_{N-1}, \\
x_{0} & =\chi_{0},
\end{aligned}
$$

and

$$
\begin{aligned}
J(u)=\sum_{k=0}^{N-1}\left\{\frac{1}{2} x_{k}^{T} Q\right. & \left(x_{k}, u_{k}, k\right) x_{k}+u_{k}^{T} P\left(x_{k}, u_{k}, k\right) x_{k} \\
& \left.+\frac{1}{2} u_{k}^{T} R\left(x_{k}, u_{k}, k\right) u_{k}+q\left(x_{k}, u_{k}, k\right)\right\}+F\left(x_{N}\right)
\end{aligned}
$$

where the symbol $T$ denotes transpose of a vector or matrix and the functions $g$ and $q$ are defined by

$$
\begin{aligned}
g(\chi, \omega, k) \triangleq & f(\chi, \omega, k)-A(\chi, \omega, k) \chi-B(\chi, \omega, k) \omega, \\
q(\chi, \omega, k) \triangleq & L(\chi, \omega, k)-\frac{1}{2} \chi^{T} Q(\chi, \omega, k) \chi \\
& -\omega^{T} P(\chi, \omega, k) \chi-\frac{1}{2} \omega^{T} R(\chi, \omega, k) \omega .
\end{aligned}
$$

If there is no confusion possible, to avoid cumbersome notation such as

$$
A\left(x_{k}, u_{k}, k\right),\left.\quad \frac{\partial f}{\partial x}(\chi, \omega, k)\right|_{\omega=u_{k}} ^{\chi=x_{k}}, \quad \text { etc. }
$$

we shall simply write

$$
A_{k}, \frac{\partial f_{k}}{\partial x}, e t c ., \text { respectively. }
$$




\section{Statement of the algorithm}

Let $\bar{x}=\left(\bar{x}_{0}, \bar{x}_{1}, \ldots, \bar{x}_{N}\right)$, the solution of (2.1) (or, equivalently of (2.3)) for some nominal control $\bar{u}=\left(\bar{u}_{0}, \bar{u}_{1}, \ldots, \bar{u}_{N-1}\right)$, denote a nominal state of the system. Define $\bar{S}_{k} \in R^{n \times n}, k \in I_{N-1}$ as the solution of the matrix difference equation

$$
\bar{S}_{k}=\bar{Q}_{k}+\bar{A}_{k}^{T} \bar{S}_{k+1} \bar{A}_{k}-\bar{W}_{k}^{T} \bar{V}_{k}^{-1} \bar{W}_{k} ; \quad \bar{S}_{N}=\bar{Q}_{N}
$$

and let

$$
\bar{h}_{k} \in R^{n}, \quad k \in I_{N-1}
$$

be the solution of

$$
\bar{h}_{k}^{T}=\bar{\phi}_{k}-\bar{\psi}_{k}^{T} \bar{V}_{k}^{-1} \bar{W}_{k}+\bar{h}_{k+1}\left(\frac{\partial \bar{f}_{k}}{\partial x}-\frac{\partial \bar{f}_{k}}{\partial u} \bar{V}_{k}^{-1} \bar{W}_{k}\right) ; \quad \bar{h}_{N}^{T}=\frac{d e\left(\bar{x}_{N}\right)}{d x}
$$

where

$$
\begin{gathered}
\bar{W}_{k} \triangleq\left(\bar{B}_{k}^{T} \bar{S}_{k+1} \bar{A}_{k}+\bar{P}_{k}\right), \\
\bar{V}_{k} \triangleq \bar{D}_{k}+\bar{B}_{k}^{T}{\overline{S_{k+1}}}_{k}, \bar{B}_{k},
\end{gathered}
$$

$\bar{D}_{k} \in R^{m \times m}$ is a positive definite matrix, and

$$
\begin{gathered}
e(\chi)=F(\chi)-\frac{1}{2} T \bar{Q}_{N} \chi \\
\bar{\phi}_{k}^{T} \triangleq \frac{\partial \bar{\rho}_{k}}{\partial x}-\bar{u}_{k}^{T} \bar{W}_{k}, \\
\bar{\psi}_{k}^{T} \triangleq \frac{\partial \bar{\rho}_{k}}{\partial u}-\bar{u}_{k}^{T} \bar{V}_{k}-\bar{x}_{k}^{T} \bar{W}_{k}, \\
\rho_{k}(\chi, \omega) \triangleq \frac{1}{2} \bar{x}_{k}^{T}\left[Q_{k}(\chi, \omega)+A_{k}^{T}(\chi, \omega) \bar{S}_{k+1} A_{k}(\chi, \omega)\right] \bar{x}_{k} \\
+\omega^{T}\left[B_{k}^{T}(\chi, \omega) \bar{S}_{k+1} A_{k}(\chi, \omega)+P_{k}(\chi, \omega)\right] \chi \\
+\frac{1}{2} \omega^{T}\left[R_{k}(\chi, \omega)+B_{k}^{T}(\chi, \omega) \bar{S}_{k+1} B_{k}(\chi, \omega)\right] \omega \\
+g_{k}^{T}(\chi, \omega) \bar{S}_{k+1}\left[A_{k}(\chi, \omega) \chi+B_{k}(\chi, \omega) \omega+g_{k}(\chi, \omega)\right] \\
+q_{k}(\chi, \omega) .
\end{gathered}
$$

The bars above the symbols indicate that the respective quantities are evaluated at $\chi=\bar{x}_{k}$ and $\omega=\bar{u}_{k}$. Also $A_{k}(\chi, \omega)$, etc., denote $A(\chi, \omega, k)$, etc., $\partial \bar{f}_{k} / \partial x$ and $\partial \bar{f}_{k} / \partial u$ denote the Jacobians, whilst $\partial \bar{\rho}_{k} / \partial x$ and $\partial \bar{\rho}_{k} / \partial u$ are considered row vectors. Observe that, for $\bar{D}_{k}>0, \bar{V}_{k}>0$.

In addition, define

$$
\begin{gathered}
\bar{\tau}_{k} \triangleq \bar{u}_{k}+\bar{V}_{k}^{-1} \bar{W}_{k} \bar{x}_{k}, \\
\bar{\beta}_{k}^{r} \triangleq \bar{\psi}_{k}^{T} \bar{V}_{k}^{-1}+\bar{h}_{k+1}^{r} \frac{\partial \bar{f}_{k}}{\partial u} \bar{V}_{k}^{-1}+\bar{\tau}_{k}
\end{gathered}
$$


and

$$
\Delta(\bar{u}) \triangleq \sum_{k=0}^{N-1}\left\{\bar{\beta}_{k}^{T} \bar{V}_{k} \bar{\beta}_{k}\right\} .
$$

Then the proposed algorithm can be stated as follows.

Discrete time LQRE algorithm

STEP 1 . Select some nominal control $\bar{u}$, compute the nominal state $\bar{x}$ according to $(2.1)$ and the value of the cost functional $J(\bar{u})$ for that nominal control, given by $(2.2)$.

STEP 2. Solve equations (3.1) and (3.2). Compute $\Delta(\bar{u})$ according to (3.11). Set $\varepsilon=1$. If $\Delta(\bar{u})=0$ stop.

STEP 3. Apply control $\tilde{u}: \tilde{u}_{k}=\bar{u}_{k}+\delta u_{k}, k \in I_{N-1}$, and compute the corresponding state of the system $\tilde{x}: \tilde{x}_{k}=\bar{x}_{k}+\delta x_{k}, k \in I_{N} \triangleq\{0,1, \ldots, N\}$, where $\delta u_{k}=-\varepsilon \bar{\beta}_{k}-\bar{V}_{k}^{-1} \bar{W}_{k} \delta x_{k}$. Compute the cost $J(\tilde{u})$.

STEP 4. If $J(\tilde{u})-J(\bar{u})+\frac{1}{2} \varepsilon \Delta(\bar{u})<0$, set $\bar{x}=\tilde{x}, \bar{u}=\tilde{u}, \bar{u}=\tilde{u}, J(\bar{u})=J(\tilde{u})$ and go to Step 2. If the above criterion is not satisfied, set $\varepsilon$ to $\varepsilon / 2$ and go to Step 3.

REMARK 3.1. Although the proofs in Sections 4, 5 and 6 hold for an arbitrary $D_{k}>0$, unless otherwise stated, it will be assumed that $\bar{D}_{k}=\bar{R}_{k}$.

REMARK 3.2. All continuous-time LQRE algorithms presented in [6] have their discrete-time analogs. Both first and second order algorithms can be obtained depending on the manner in which $A_{k}, B_{k}, Q_{k}, P_{k}, B_{k}$ and $Q_{N}$ are chosen. An algorithm worth explicit mention is the discrete-time analog of FORE-3 [6], which is a highly efficient first-order algorithm devised specifically for problems with nonlinear dynamics and quadratic cost; in the discrete version of FORE-3 we set $A_{k}=\partial f u_{k} / \partial x, B_{k}=\partial \bar{f}_{k} / \partial u\left(Q_{k}>0, P_{k}, R_{k}>0\right.$ and $Q_{N}>0$ are given when the problem is specified).

\section{Proof of reduction at each iteration}

In this section we derive an expression (correct to the first order of $\delta x_{k}$ and $\delta u_{k}$ ) for the increment of the cost functional at each iteration of the proposed algorithm and show that the increment represents a reduction in the value of the cost functional.

From the identity

$$
\sum_{k=0}^{N-1}\left\{\frac{1}{2}\left(x_{k+1}^{T} \bar{S}_{k+1} x_{k+1}-x_{k}^{T} \bar{S}_{k} x_{k}\right)\right\}-\frac{1}{2} x_{N}^{T} \bar{S}_{N} x_{N}+\frac{1}{2} x_{0}^{T} \bar{S}_{0} x_{0}=0
$$


after $x_{k+1}$ and $\bar{S}_{k}$ are substituted with the right-hand sides of (2.3) and (3.1), it follows that

$$
\begin{array}{r}
\sum_{k=0}^{N-1} \frac{1}{2}\left\{\left(A_{k} x_{k}+B_{k} u_{k}+g_{k}\right)^{T} \bar{S}_{k+1}\left(A_{k} x_{k}+B_{k} u_{k}+g_{k}\right)\right. \\
\left.-x_{k}^{T}\left(\bar{Q}_{k}+\bar{A}_{k} \bar{S}_{k+1} \bar{A}_{k}-\bar{W}_{k}^{T} \bar{V}_{k}^{-1} \bar{W}_{k}\right) x_{k}\right\} \\
-\frac{1}{2} x_{N}^{T} \bar{Q}_{N} x_{N}+\frac{1}{2} \chi_{0}^{T} \bar{S}_{0} \chi_{0}=0 .
\end{array}
$$

Adding this zero quantity to the expression (2.4) we have

$$
\begin{aligned}
J(u)=\sum_{k=0}^{N-1}\left\{\frac{1}{2} x_{k}^{T} A_{k}^{T} \bar{S}_{k+1} A_{k} x_{k}+\frac{1}{2} x_{k}^{T} A_{k}^{T} \bar{S}_{k+1} B_{k} u_{k}+\frac{1}{2} u_{k}\right. \\
+g_{k}^{T} \bar{S}_{k+1} A_{k} x_{k}+g_{k}^{T} \bar{S}_{k+1} B_{k} u_{k}+\frac{1}{2} g_{k}^{T} \bar{S}_{k+1} g_{k} \\
\quad-\frac{1}{2} x_{k}^{T}\left(\left(\bar{Q}_{k}+\bar{A}_{k} \bar{S}_{k+1} \bar{A}_{k}\right)-\bar{W}_{k}^{T} \bar{V}_{k}^{-1} \bar{W}_{k}\right) x_{k} \\
\left.\quad+\frac{1}{2} x_{k}^{T} Q_{k} x_{k}+u_{k}^{T} P_{k} x_{k}+\frac{1}{2} u_{k}^{T} R_{k} u_{k}+q_{k}\right\}+\frac{1}{2} \chi_{0} \bar{S}_{0} \chi_{0}+e\left(x_{N}\right)
\end{aligned}
$$

where $e$ is defined by (3.5).

Consequently,

$$
\begin{aligned}
& J(\bar{u})=\frac{1}{2} \chi_{0}^{T} \bar{S}_{0} \chi_{0}+e\left(\bar{x}_{N}\right) \\
& +\sum_{k=0}^{N-1}\left\{\frac{1}{2} \bar{u}_{k}^{T} \bar{R}_{k} \bar{u}_{k}+\bar{u}_{k}^{T} \bar{B}_{k}^{T} \bar{S}_{k+1} \bar{A}_{k} \bar{x}_{k}+\bar{u}_{k}^{T} \bar{P}_{k} \bar{x}_{k}\right. \\
& +\frac{1}{2} \bar{u}_{k}^{T} \bar{B}_{k}^{T} \bar{S}_{k+1} \bar{B}_{k} \bar{u}_{k}+\bar{g}_{k}^{T} \bar{S}_{k+1} \bar{A}_{k} \bar{x}_{k}+\bar{g}_{k}^{T} \bar{S}_{k+1} \bar{B}_{k} \bar{u}_{k} \\
& \left.+\frac{1}{2} \bar{g}_{k}^{T} \bar{S}_{k+1} \bar{g}_{k}+\bar{q}_{k}+\frac{1}{2} \bar{x}_{k}^{T} \bar{W}_{k}^{T} \bar{V}_{k}^{-1} \bar{W}_{k} \bar{x}_{k}\right\}
\end{aligned}
$$

and

$$
\begin{aligned}
& J(\tilde{u})=\frac{1}{2} \chi_{0}^{T} \bar{S}_{0} \chi_{0}+e\left(\tilde{x}_{N}\right) \\
& +\sum_{k=0}^{N-1}\left\{\frac{1}{2} \tilde{x}_{k}^{T} \tilde{Q}_{k} \tilde{x}_{k}-\frac{1}{2} \tilde{x}_{k}^{T} \bar{Q}_{k} \tilde{x}_{k}+\frac{1}{2} \tilde{x}_{k}^{T} \tilde{A}_{k}^{T} \bar{S}_{k+1} \tilde{A}_{k} \tilde{x}_{k}\right. \\
& \quad-\frac{1}{2} \tilde{x}_{k}^{T} \bar{A}_{k}^{T} \bar{S}_{k+1} \bar{A}_{k} x+\frac{1}{2} \tilde{u}_{k}^{T} \tilde{R}_{k} \tilde{u}_{k}+\tilde{u}_{k}^{T} \tilde{B}_{k} \bar{S}_{k+1} \tilde{A}_{k} \tilde{x}_{k} \\
& +\quad \tilde{u}_{k}^{T} \tilde{P}_{k} \tilde{x}_{k}+\frac{1}{2} \tilde{u}_{k}^{T} \tilde{B}_{k}^{T} \bar{S}_{k+1} \tilde{B}_{k} \tilde{u}_{k}+\tilde{g}_{k}^{T} \bar{S}_{k+1} \tilde{A}_{k} \tilde{x}_{k} \\
& + \\
& \left.+\tilde{g}_{k}^{T} \bar{S}_{k+1} \tilde{B}_{k} \tilde{u}_{k}+\frac{1}{2} \tilde{g}_{k}^{T} \bar{S}_{k+1} \tilde{g}_{k}+\tilde{q}_{k}+\frac{1}{2} \tilde{x}_{k}^{T} \bar{W}_{k}^{T} \bar{V}_{k}^{-1} \bar{W}_{k} \tilde{x}_{k}\right\}
\end{aligned}
$$


where $\tilde{A}_{k}, \tilde{B}_{k}$, etc., stand for $A_{k}\left(\tilde{x}_{k}, \tilde{u}_{k}\right), B_{k}\left(\tilde{x}_{k}, \tilde{u}_{k}\right)$, etc., respectively. It follows that

$$
\begin{aligned}
\delta J=J(\tilde{u})-J(\bar{u})=J(\bar{u}+\delta u) & -J(\bar{u}) \\
=e\left(\tilde{x}_{N}\right)-e\left(\bar{x}_{N}\right)+\sum_{k=0}^{N-1}\left\{\rho_{k}\left(\tilde{x}_{k}, \tilde{u}_{k}\right)-\rho_{k}\left(\bar{x}_{k}, \bar{u}_{k}\right)\right. & \\
& -\bar{u}_{k}^{T} \bar{V}_{k} \delta u_{k}-\bar{x}_{k}^{T} \bar{W}_{k} \delta u_{k}-\bar{u}_{k}^{T} \bar{W}_{k} \delta x_{k} \\
& +\bar{\tau}_{k}^{T} \bar{V}_{k}\left(\delta u_{k}+\bar{V}_{k}^{-1} \bar{W}_{k} \delta x_{k}\right)+\bar{z}_{k}^{T}\left[\begin{array}{cc}
Y_{k} & 0 \\
0 & 0
\end{array}\right] \delta z_{k} \\
& \left.+\frac{1}{2} \delta z_{k}^{T}\left[\begin{array}{cc}
T_{k} & 0 \\
0 & 0
\end{array}\right] \delta z_{k}\right\}
\end{aligned}
$$

where

$$
\begin{gathered}
\bar{\tau}_{k} \triangleq \bar{u}_{k}+\bar{V}_{k}^{-1} \bar{W}_{k} \bar{x}_{k}, \\
\bar{z}_{k}^{T} \triangleq\left[\bar{x}_{k}^{T} \bar{u}_{k}^{T}\right], \quad \delta z_{k}^{T} \triangleq\left[\delta x_{k}^{T} \delta u_{k}^{T}\right], \\
Y_{k} \triangleq\left(\tilde{Q}_{k}+\tilde{A}_{k} \bar{S}_{k+1} \tilde{A}_{k}\right)-\left(\bar{Q}_{k}+\bar{A}_{k} \bar{S}_{k+1} \bar{A}_{k}\right), \\
T_{k} \triangleq Y_{k}+\bar{W}_{k}^{T} \bar{V}_{k}^{-1} \bar{W}_{k}
\end{gathered}
$$

and $\rho_{k}, k \in I_{N-1}$ is a scalar function on $R^{n} \times R^{m}$ defined by (3.8).

As $A_{k}$ and $Q_{k}$ are continuous in both arguments, $Y_{k}$ converges to 0 as $\left|\delta z_{k}\right| \rightarrow 0$, where || is the Euclidean norm in $R^{n+m}$. Expanding $\rho_{k}\left(\tilde{x}_{k}, \tilde{u}_{k}\right)=$ $\rho_{k}\left(\bar{x}_{k}+\delta x_{k}, \bar{u}_{k}+\delta u_{k}\right)$ about $\bar{x}_{k}$ and $\bar{u}_{k}$ in $\delta x_{k}$ and $\delta u_{k}$, we have

$$
\begin{aligned}
\delta J= & e\left(\tilde{x}_{N}\right)-e\left(\bar{x}_{N}\right)+\sum_{k=0}^{N-1}\left\{\left(\frac{\partial \bar{\rho}_{k}}{\partial u}-\bar{u}_{k}^{T} \bar{V}_{k}-\bar{x}_{k}^{T} \bar{W}_{k}\right) \delta u_{k}\right. \\
& \left.+\left(\frac{\partial \bar{\rho}_{k}}{\partial x}-\bar{u}_{k}^{T} \bar{W}_{k}\right) \delta x_{k}+\bar{\tau}_{k}^{T} \bar{V}_{k} \delta u_{k}+\bar{\tau}_{k}^{T} \bar{W}_{k} \delta x_{k}\right\} \\
& +\sum_{k=0}^{N-1} \sigma_{k}\left(\delta z_{k}\right)
\end{aligned}
$$

where

$$
\sigma_{k}\left(\delta z_{k}\right) \triangleq \bar{z}_{k}^{T}\left[\begin{array}{cc}
Y_{k} & 0 \\
0 & 0
\end{array}\right] \delta z_{k}+\frac{1}{2} \delta z_{k}^{T}\left[\begin{array}{cc}
T_{k} & 0 \\
0 & 0
\end{array}\right] \delta z_{k}+\theta_{k}\left(\delta z_{k}\right)
$$

and

$$
\lim _{\left|\delta z_{k}\right| \rightarrow 0} \frac{\left|\theta_{k}\left(\delta z_{k}\right)\right|}{\left|\delta z_{k}\right|}=0 .
$$


Clearly,

$$
\lim _{\left|\delta z_{k}\right| \rightarrow 0} \frac{\left|\sigma_{k}\left(\delta z_{k}\right)\right|}{\left|\delta z_{k}\right|}=0 .
$$

Further by expanding $e\left(\tilde{x}_{N}\right)$ about $\bar{x}_{N}$ in $\delta x_{N},(4.2)$ can be written in the form

$$
\begin{aligned}
\delta J= & \frac{\operatorname{de}\left(\bar{x}_{N}\right)}{d x} \delta x_{N}+\sigma_{N}\left(\delta x_{N}\right) \\
& +\sum_{k=0}^{N-1}\left\{\bar{\psi}_{k}^{T} \delta u_{k}+\bar{\phi}_{k}^{T} \delta x_{k}+\bar{\tau}_{k}^{T} \bar{V}_{k} \delta u_{k}+\bar{\tau}_{k}^{T} \bar{W}_{k} \delta x_{k}\right\}+\sum_{k=0}^{N-1} \sigma_{k}\left(\delta z_{k}\right)
\end{aligned}
$$

where $\bar{\phi}_{k}$ and $\bar{\psi}_{k}$ are defined by (3.6) and (3.7), respectively and $\lim _{\left|\delta z_{N}\right| \rightarrow 0}\left|\sigma_{N}\left(\delta z_{N}\right)\right| /\left|\delta z_{N}\right|=0$.

On the other hand if $\bar{h}_{k} \in R^{n}$ is defined as the solution of (3.2) and $\delta x_{k}$ is considered as the solution of

$$
\delta x_{k+1}=\frac{\partial \bar{f}_{k}}{\partial x} \delta x_{k}+\frac{\partial \bar{f}_{k}}{\partial u} \delta u_{k}+\eta_{k}\left(\delta z_{k}\right), \quad k \in I_{N-1}, \quad \delta x_{0}=0,
$$

where $\lim _{\left|\delta z_{k}\right| \rightarrow 0}\left|\eta_{k}\left(\delta z_{k}\right)\right| /\left|\delta z_{k}\right|=0$, then, from the identity

$$
\bar{h}_{0}^{T} \delta x_{0}-\bar{h}_{N}^{T} \delta x_{N}+\sum_{k=0}^{N-1}\left(\bar{h}_{k}^{T} \delta x_{k}-\bar{h}_{k+1}^{T} \delta x_{k+1}\right)=0,
$$

it follows that

$$
\begin{array}{r}
\frac{-d e\left(\bar{x}^{N}\right)}{d x} \delta x_{N}+\sum_{k=0}^{N-1}\left\{-\bar{\phi}_{k}^{T}+\bar{\psi}_{k}^{T} \bar{V}_{k}^{-1} \bar{W}_{k}+\bar{h}_{k+1}^{T}\left(-\frac{\partial \bar{f}_{k}}{\partial x}+\frac{\partial \bar{f}_{k}}{\partial u} \bar{V}_{k}^{-1} \bar{W}_{k}\right) \delta x_{k}\right. \\
\left.+\bar{h}_{k+1}^{T}\left(\frac{\partial \bar{f}_{k}}{\partial x} \delta x_{k}+\frac{\partial \bar{f}_{k}}{\partial u} \delta u_{k}+\eta_{k}\left(\delta z_{k}\right)\right)\right\}=0 .
\end{array}
$$

Adding (4.5) and (4.3), the expression for $\delta J$ becomes

$$
\begin{aligned}
\delta J=\sum_{k=0}^{N-1}\{ & \bar{\psi}_{k}^{T} \delta u_{k}+\bar{\psi}_{k}^{T} \bar{V}_{k}^{-1} \bar{W}_{k} \delta x_{k}+\bar{h}_{k+1}^{T} \frac{\partial \bar{f}_{k}}{\partial u} \delta u_{k} \\
& \left.+\bar{h}_{k+1}^{T} \frac{\partial \bar{f}_{k}}{\partial u} \bar{V}_{k}^{-1} \bar{W}_{k} \delta x_{k}+\bar{\tau}_{k}^{T} \bar{V}_{k} \delta u_{k}+\bar{\tau}_{k}^{T} \bar{W}_{k} \delta x_{k}\right\}+\left(\sum_{k=0}^{N-1} r_{k}\right)+\sigma_{N}\left(\delta z_{N}\right)
\end{aligned}
$$

where $r_{k} \triangleq \sigma_{k}\left(\delta z_{k}\right)+\bar{h}_{k+1}^{T} \eta_{k}\left(\delta z_{k}\right)$. Clearly, $\lim _{\left|\delta z_{k}\right| \rightarrow 0}\left|r_{k}\right| /\left|\delta z_{k}\right|=0$. Let

$$
\bar{\beta}_{k}^{T} \triangleq \bar{\psi}_{k}^{T} \bar{V}_{k}^{-1}+\bar{h}_{k+1}^{T} \frac{\partial \tilde{f}_{k}}{\partial u} \bar{V}_{k}^{-1}+\bar{\tau}_{k}^{T}
$$

and

$$
r \triangleq \sum_{k=0}^{N-1} r_{k}+\sigma_{N}\left(\delta z_{N}\right)
$$


Then

$$
\delta J=\sum_{k=0}^{N-1}\left\{\bar{\beta}_{k}^{T} \bar{V}_{k}\left(\delta u_{k}+\bar{V}_{k}^{-1} \bar{W}_{k} \delta x_{k}\right)\right\}+r
$$

Finally, by setting

$$
\delta u_{k}=-\varepsilon \bar{\beta}_{k}-\bar{V}_{k}^{-1} \bar{W}_{k} \delta x_{k}, \quad \varepsilon \in[0,1],
$$

we have

$$
\begin{aligned}
& \delta J=-\varepsilon \sum_{k=0}^{N-1}\left\{\bar{\beta}_{k}^{T} \bar{V}_{k} \bar{\beta}_{k}\right\}+r, \\
& \delta J=-\varepsilon \Delta(\bar{u})+r
\end{aligned}
$$

where

$$
\Delta(\bar{u}) \triangleq \sum_{k=0}^{N-1} \bar{\beta}_{k}^{T} \bar{V}_{k} \bar{\beta}_{k} .
$$

If $\delta u_{k}$ is chosen according to (4.7), $\delta x_{k}$, the solution of

$$
\bar{x}_{k+1}+\delta x_{k+1}=f_{k}\left(\bar{x}_{k}+\delta x_{k}, \bar{u}_{k}+\delta u_{k}\right), \quad k \in I_{N-1}, \delta x_{0}=0,
$$

with $\bar{x}_{k}, k \in I_{N}=\{0,1, \ldots, N\}$ and $\bar{u}_{k}, k \in I_{N-1}$, specified, is a continuous function of $\varepsilon$. Therefore, arguing along the lines similar to those in [6] for the continuous case, it is not difficult to show that $\lim _{\varepsilon \rightarrow 0}|r| / \varepsilon=0$.

Since $\bar{V}_{k}$ is positive definite it is immediate from (4.8) that for $\bar{\beta}_{k} \neq 0$ and $\varepsilon$ sufficiently small,

$$
\delta J=-\varepsilon \sum_{k=0}^{N-1} \bar{\beta}_{k}^{T} \bar{V}_{k} \bar{\beta}_{k}+r<0,
$$

i.e. under the above assumptions the variation $\delta J$ represents a reduction in the value of the cost functional. Clearly,

$$
\sum_{k=0}^{N-1} \bar{\beta}_{k}^{T} \bar{V}_{k} \bar{\beta}_{k}=0
$$

or, equivalently,

$$
\vec{\beta}_{k}=0, \quad k \in I_{N-1}
$$

are necessary conditions for optimality. In the sequel any control which satisfies (4.12) will be referred to as a desirable control or desirable point. (Note that if $\bar{\beta}_{k}=0$ for $k \in I_{N-1}$ is not satisfied, $J(u)$ can be further reduced by the LQRE 
algorithm.) In Section 5, we shall show that the above condition is equivalent to the first order necessary condition $\partial H_{k} / \partial u=0, k \in I_{N-1}$.

REMARK 4.1. Using similar arguments to those in [6], page 877, it can easily be shown that the discrete-time LQRE algorithm converges in one step on the linear-quadratic problem defined in [1], page 42.

REMARK 4.2. Similarly, it can be shown that the discrete-time LQRE algorithm exhibits one step convergence on the problem with the system dynamics and cost functional given by

$$
\begin{aligned}
x_{k+1}= & A_{k} x_{k}+B_{k} u_{k}+a_{k}, \quad k \in I_{N-1} ; x_{0}=x_{0}, \\
J(u)= & \sum_{k=0}^{N-1}\left\{\frac{1}{2} x_{k}^{T} Q_{k} x_{k}+u_{k}^{T} P_{k} x_{k}+\frac{1}{2} u_{k}^{T} R_{k} u_{k}+b_{k}^{T} x_{k}+c_{k}^{T} u_{k}+d_{k}\right\} \\
& +\frac{1}{2} x_{N}^{T} Q_{N} x_{N}+p_{N}^{T} x_{N}+s_{N},
\end{aligned}
$$

where $A_{k}, B_{k}, Q_{k} \geqslant 0, P_{k}, R_{k}>0, Q_{N} \geqslant 0, a_{k}, b_{k}, c_{k}, d_{k}, p_{N}$ and $s_{N}$ are constants (independent of $x_{k}$ and $u_{k}$ ) of appropriate dimensions.

\section{Criterion for optimality}

For convenience "bars" above symbols will be dropped in this section. The control $u$,

$$
u^{T}=\left[u_{0}^{T} u_{1}^{T} \cdots u_{N-1}^{T}\right]=\left[u_{0}^{1} \cdots u_{0}^{m} u_{1}^{1} \cdots u_{1}^{m} \cdots u_{N-1}^{1} \cdots u_{N-1}^{m}\right]
$$

and the state of the system $x$,

$$
x^{T}=\left[x_{0}^{T} x_{1}^{T} \cdots x_{N}^{T}\right]=\left[x_{0}^{1} \cdots x_{0}^{n} x_{1}^{1} \cdots x_{1}^{n} \cdots x_{N}^{1} \cdots x_{N}^{n}\right]
$$

can be considered as points in $R^{(N-1) m}$ and $R^{N n}$. From the equation

$$
\begin{aligned}
x_{k} & =f_{k-1}\left(x_{k-1}, u_{k-1}\right)=f_{k-1}\left(f_{k-2}\left(x_{k-2}, u_{k-2}\right), u_{k-1}\right) \\
& =f_{k-1} f_{k-2}\left(\ldots, u_{k-2}, u_{k-1}\right)
\end{aligned}
$$

and from the assumptions concerning the differentiability of $f_{k}, k \in I_{N-1}$, it follows then that $x_{k}$ is differentiable with respect to $u_{0}, u_{1}, \ldots, u_{k-1}$ which, in turn, implies that $x_{k}$ and $x$ are differentiable in $u$. (Here $\partial x_{k+1} / \partial u_{i}=0, k<i \leqslant$ $N-1$.

Thus, $J$ given by (2.2) is differentiable in $u \in R^{N m}$ and, consequently, in the discrete-time case the Gateaux and Fréchet differentials coincide with ordinary 
differentials. Therefore, the first order necessary condition for optimality of discrete-time systems can be stated as $d J(u) / d u=0$ or in an equivalent form $\left(\partial H_{k} / \partial u\right)\left(x_{k}, u_{k}, \lambda_{k+1}\right)=0$, where $H_{k}\left(\chi, \omega, \lambda_{k+1}\right) \triangleq L_{k}(\chi, \omega)+\lambda_{k+1}^{T} f_{k}(\chi, \omega)$ and $\lambda_{k}$ is the solution of the difference equation $\lambda_{k}^{T}=\left(\partial H_{k} / \partial x\right)\left(x_{k}, u_{k}, \lambda_{k+1}\right)$, $k \in I_{N-1}, \lambda_{N}^{T}=\partial F\left(x_{N}\right) / \partial x(c f .[1]$, page 67).

Let $\nu_{k}$ be the solution of the difference equation

$$
\nu_{k}^{T}=\phi_{k}^{T}+\tau_{k}^{T} W_{k}+v_{k+1}^{T} \frac{\partial f_{k}}{\partial x}, \quad k \in I_{N-1}, \nu_{N}^{T}=\frac{d e\left(X_{N}\right)}{d x} .
$$

As $\delta x_{k}$ is the solution of (4.4), from the identity

$$
\nu_{0}^{T} \delta x_{0}-\nu_{N}^{T} \delta x_{N}+\sum_{k=0}^{N-1}\left(\nu_{k+1}^{T} \delta x_{k+1}-\nu_{k}^{T} \delta x_{k}\right)=0
$$

it follows that

$$
\begin{aligned}
&-\nu_{N}^{T} \delta x_{N}+\sum_{k=0}^{N-1}\left[\nu _ { k + 1 } ^ { T } \left(\frac{\partial f_{k}}{\partial x} \delta\right.\right.\left.x_{k}+\frac{\partial f_{k}}{\partial u} \delta u_{k}+\eta_{k}\right) \\
&\left.-\left(\phi_{k}^{T}+\tau_{k}^{T} W_{k}+\nu_{k+1}^{T} \frac{\partial f_{k}}{\partial x}\right) \delta x_{k}\right]=0 .
\end{aligned}
$$

Adding this to the expression (4.3) for $\delta J$, gives

$$
\delta J=\sigma_{N}\left(\delta x_{N}\right)+\sum_{k=0}^{N-1}\left[\nu_{k+1}^{T} \frac{\partial f_{k}}{\partial u}+\psi_{k}^{T}+\tau_{k}^{T} V_{k}\right] \delta u_{k}+\sum_{k=0}^{N-1}\left[\nu_{k+1}^{T} \eta_{k}+\sigma_{k}\left(\delta z_{k}\right)\right]
$$

or

$$
\delta J=\sum_{k=0}^{N-1}\left[\xi_{k}^{r} \delta u_{k}+\alpha_{k}\left(\delta z_{k}\right)\right]+\sigma_{N}\left(\delta x_{N}\right)
$$

where

$$
\xi_{k}^{T} \triangleq \nu_{k+1}^{T} \frac{\partial f_{k}}{\partial u}+\psi_{k}^{T}+\tau_{k}^{T} V_{k}
$$

and

$$
\alpha_{k}\left(\delta z_{k}\right) \triangleq \nu_{k+1}^{T} \eta_{k}\left(\delta z_{k}\right)+\sigma_{k}\left(\delta z_{k}\right) .
$$

Since $\lim _{|\delta u| \rightarrow 0}\left|\alpha_{k}\left(z_{k}\right)\right| /|\delta u|=0$ (cf. (4.2), (4.3) and (4.4)) and the expansion

$$
\delta J=\sum_{k=0}^{N-1}\left(\frac{\partial H_{k}\left(x_{k}, u_{k}, \lambda_{(k+1)}\right)}{\partial u}\right) \delta u_{k}
$$

(correct to the first order of $\delta u$ ) is unique. It follows that

$$
\xi_{k}^{T}=\frac{\partial H_{k}\left(x_{k}, u_{k}, \lambda_{k+1}\right)}{\partial u}
$$


THEOREM 1. For the discrete-time control problem defined in Section 2, the optimality criterion $\beta_{k}=0$ is equivalent to the criterion

$$
\frac{\partial H_{k}}{\partial u} \triangleq \nu_{k+1}^{T} \frac{\partial f_{k}}{\partial u}+\psi_{k}^{T}+\tau_{k}^{T} V_{k}=0, \quad k \in I_{N-1} .
$$

Proof. As $V_{k}>0$,

$$
\beta_{k}^{T}=\psi_{k}^{T} V_{k}^{-1}+h_{k+1}^{T} \frac{\partial f_{k}}{\partial u} V_{k}^{-1}+\tau_{k}^{T}=0
$$

yields

$$
\psi_{k}^{T}+h_{k+1}^{T} \frac{\partial f_{k}}{\partial u}+\tau_{k}^{T} V_{k}=0
$$

with $h_{k}$ defined by (3.2). From (5.2),

$$
-\tau_{k}^{T} W_{k}=\psi_{k}^{T} V_{k}^{-1} W_{k}+h_{k+1}^{T} \frac{\partial f_{k}}{\partial u} V_{k}^{-1} W_{k}
$$

which implies that (3.2) can be written in the form

$$
h_{k}^{T}=\phi_{k}^{T}+\tau_{k}^{T} W_{k}+h_{k+1}^{T} \frac{\partial f_{k}}{\partial x}, \quad k \in I_{N-1}, h_{N}^{T}=\frac{d e\left(x_{N}\right)}{d x} .
$$

Comparing this with (5.1), we see that in this case $h_{k}=\nu_{k}$ and, consequently, $\partial H_{k} / \partial u=0$ follows from (5.2). On the other hand if $\partial H_{k} / \partial u=\nu_{k+1}^{T} \partial f_{k} / \partial u+\psi_{k}^{T}$ $+\tau_{k}^{T} V_{k}=0$ is satisfied, $\tau_{k} W_{k}=-\nu_{k+1}^{T}\left(\partial f_{k} / \partial u\right) V_{k}^{-1} W_{k}-\psi_{k}^{T} V_{k}^{-1} W_{k}$ and (5.1) can be written in the form

$$
\nu_{k}^{T}=\phi_{k}^{T}-\psi_{k}^{T} V_{k}^{-1} W_{k}+\nu_{k+1}^{T}\left(\frac{\partial f_{k}}{\partial x}-\frac{\partial f_{k}}{\partial u} V_{k}^{-1} W_{k}\right) .
$$

Comparing this with (3.2), it follows (by inspection) that in this case $\nu_{k}=h_{k}$. Thus (5.2) follows from $\partial H_{k} / \partial u=0$. Since we have proved that both $\beta_{k}=0$, $k \in I_{N-1}$ implies $\partial H_{k} / \partial u=0, k \in I_{N-1}$ and $\partial H_{k} / \partial u=0, k \in I_{N-1}$ implies $\beta_{k}=0, k \in I_{N-1}$, the two criteria are equivalent.

\section{Proof of convergence}

The LQRE algorithm for discrete-time systems utilizes the search function $\alpha$ : $U \rightarrow U$ defined by

$$
u^{T}(i+1)=\alpha^{T}(u(i))=\left[\alpha_{0}^{T}\left(u_{0}(i)\right) \cdots \alpha_{N-1}^{T}\left(u_{N-1}(i)\right)\right]
$$

where $i$ denotes the iteration number and

$$
\alpha_{k}\left(u_{k}(i)\right)=u_{k}(i)-\varepsilon(i) \beta_{k}(i)-V_{k}^{-1}(i) W_{k}(i) \delta x_{k}(i), \quad k \in I_{N-1} .
$$

Define $x_{k}^{0}$ as the solution of

$$
x_{k+1}^{0}=x_{k}^{0}+L_{k}\left(x_{k}, u_{k}\right)+F\left(f_{k}\left(x_{k}, u_{k}\right)\right)-F\left(x_{k}\right), \quad k \in I_{N-1}, x_{0}^{0}=F\left(x_{0}\right),
$$


and let

$$
\begin{aligned}
& y^{T} \triangleq\left[y_{0} \cdots y_{N}\right], \quad y_{k} \triangleq \beta_{k}^{T} V_{k} \beta_{k}, \quad k \in I_{N-1}, \\
& \check{x}_{k}^{T}=\left[x_{k}^{0} x_{k}^{T}\right], \quad k \in I_{N}, \\
& \check{x}^{T}=\left[\check{x}_{0}^{T} \breve{x}_{1}^{T} \cdots \check{x}_{N}^{T}\right], \quad \check{x}_{0}=\left[F\left(\chi_{0}\right) \chi_{0}\right] \\
& f_{k}^{0}\left(\check{x}_{k}, u_{k}\right) \triangleq x_{k}^{0}+L_{k}\left(x_{k}, u_{k}\right)+F\left(f_{k}\left(x_{k}, u_{k}\right)\right)-F\left(x_{k}\right), \quad k \in I_{N}, \\
& \check{f}_{k}^{T}\left(\check{x}_{k}, u_{k}\right)=\left[f_{k}^{0}\left(\check{x}_{k}, u_{k}\right) f_{k}^{T}\left(x_{k}, u_{k}\right)\right], \quad k \in I_{N} .
\end{aligned}
$$

Then on the assumption that the increment of the control is in the form (4.7), it follows that

$$
\breve{x}_{k+1}=\breve{f}_{k}\left(\check{x}_{k}, \bar{u}_{k}-\varepsilon \bar{\beta}_{k}-\bar{V}_{k}^{-1} \bar{W}_{k} \delta x_{k}\right), \quad k \in I_{N-1} ; \check{x}_{0}=\check{x}_{0} .
$$

With the above definitions, the convergence proof for the discrete case is a verbatim repetition of the convergence analysis for continuous time systems in Appendix III of [6], provided that

(i) $U=\mathscr{L}_{\infty}[0,1]$ and $W$ in [6] are formally replaced with $R^{N m}$,

(ii) $\mathscr{L}_{\infty}^{n}[0,1]$ is replaced with $R^{(N+1) n}$,

(iii) $\mathscr{L}_{\infty}^{n \times n}[0,1]$ is replaced with $R^{(N+1)(n \times n)}$,

(iv) $C[0,1]$ with $R^{N+1}$,

(v) \|\|$_{\infty}$ with $\mid$,

(vii) $\bar{R}, \bar{B} T S, \bar{\beta}_{1}$ are replaced with $\bar{V}_{k}, \bar{W}_{k}, \bar{\beta}_{k}$,

and, in general, the value $v(t)$ of any function $v$ is replaced with its discrete-time counterpart $v_{k}$.

Note also that all the Fréchet derivatives referred to in Appendix III of [6], reduce to ordinary derivatives in the discrete case.

Thus, Theorem 1 in [6] holds for the discrete-time problem and the sequence $\{u(i)\}$ constructed by the discrete-time LQRE algorithm is either finite and its last element is desirable or else it is infinite and any accumulation pont of $\{u(i)\}$ is desirable. Under the assumption

(H1) If $\{u(i)\}$ is any sequence of admissible controls and $|u(i)| \rightarrow \infty$, then $J(u(i)) \rightarrow \infty$

the sequence $\{u(i)\}$ generated by the algorithm is bounded. Suppose that the opposite is true, i.e. that the generated sequence $\{u(i)\}$ is not bounded. Then there exists a subsequence $\left\{u\left(i_{k}\right)\right\}$ of $\{u(i)\}$ such that $\left|u\left(i_{k}\right)\right| \rightarrow \infty$ and, by virtue of $(\mathrm{H} 1), J\left(u\left(i_{k}\right)\right) \rightarrow \infty$ which contradicts the fact that $\{J(u(i))\}$ is a monotone decreasing sequence as shown in Section 4. Thus, the generated sequence $\{u(i)\}$ must be bounded and there exists a closed bounded subset $\Omega$ of $U=R^{N m}$ such 
that $u(i) \in \Omega$ for all $i$. Since the set $\Omega$ is sequentially compact, the existence of accumulation points of the infinite sequence $\{u(i)\}$ is guaranteed and, consequently, the convergence of the algorithm is proved.

\section{Problems with constraints}

Consider the problem defined in Section 2, i.e.

$$
\begin{aligned}
& \text { minimise } J(u)=\sum_{k=0}^{N-1}\left\{L_{k}\left(x_{k}, u_{k}\right)+F\left(x_{N}\right)\right\}, \\
& x_{k+1}=f_{k}\left(x_{k}, u_{k}, u_{k}\right), \quad k \in I_{N-1}, x_{0}=\chi_{0},
\end{aligned}
$$

subject to the constraint

$$
G_{k}\left(x_{k}, u_{k}\right)=0, \quad k \in I_{N-1},
$$

where $G_{k}: R^{n} \times R^{m} \rightarrow R^{p}$ is continuously differentiable in both arguments. It is assumed that an optimal solution exists. The problem can be solved by adding the penalty function $\frac{1}{2} \mathscr{K}_{i} \sum_{k=0}^{N-1} G_{k}\left(x_{k}, u_{k}\right) G_{k}\left(x_{k}, u_{k}\right)$ to the cost functional and solving progressively the unconstrained approximating problem

minimise $J_{a}\left(u, K_{i}\right)=\sum_{k=0}^{N-1}\left\{L_{k}\left(x_{k}, u_{k}\right)+\frac{1}{2} \mathscr{K}_{i} G_{k}^{T}\left(x_{k}, u_{k}\right) G_{k}\left(x_{k}, u_{k}\right)\right\}+F\left(x_{N}\right)$,

$x_{k+1}=f_{k}\left(x_{k}, u_{k}\right), \quad k \in I_{N-1}, \quad x_{0}=\chi_{0}$,

for a sequence of positive scalars $\left\{\mathscr{K}_{i}\right\}$ which tends to infinity. By (5.1) $J_{a}\left(u, \mathscr{K}_{i}\right)$ can be considered as a function of $u \in R^{m N}$ and $\mathscr{K}_{i}$ only and, consequently, the results on convergence of penality function methods in [5] are directly applicable in this case.

In theory at least, then, any globally convergent method for unconstrained optimization of discrete-time systems could be used for solving the approximating problem (7.3). However, for many algorithms that might be applied, the structure of (7.3) becomes increasingly unfavourable as $\mathscr{K}_{i}$ is increased which is reflected in a poor convergence rate. In implementation of the penality function method it is, therefore, very important to select an efficient algorithm for unconstrained problems when the cost functional contains a penality term.

We shall now examine how the LQRE algorithm is affected when constraint (7.2) is linear by the addition of the penalty term to the cost functional. Linear constraints are extremely important from a practical viewpoint and they are also simplest to analyse. Assume first that the unconstrained problem (7.1) has been solved by means of the LQRE algorithm. Then, in order to solve the constrained problem by the LQRE and penalty function method, we write the cost functional 
in (7.3) in the form

$$
\begin{aligned}
J_{a}\left(u, \mathscr{K}_{i}\right)= & \sum_{k=0}^{N-1}\left\{\frac{1}{2} x_{k}^{T} Q_{k} x_{k}+u_{k}^{T} P_{k} x_{k}+\frac{1}{2} u_{k}^{T} R_{k} u_{k}+q_{k}\left(x_{k}, u_{k}\right)\right\} \\
& +\frac{1}{2} \mathscr{K}_{i}\left\{G_{k}^{T}\left(x_{k}, u_{k}\right) G_{k}\left(x_{k}, u_{k}\right)\right\}+F\left(x_{N}\right)
\end{aligned}
$$

(cf. 2.4) where $Q_{k} \geqslant 0, R_{k}>0$ denote the matrices used for solving the unconstrained problem. (It is assumed that $D_{k}=R_{k}$; $c f$. (3.4) and Remark 3.1.) As the constraints are linear, we have

$$
G_{k}\left(x_{k}, u_{k}\right) \triangleq\left[\Gamma_{k} \Pi_{k}\right]\left[\begin{array}{l}
x_{k} \\
u_{k}
\end{array}\right]-\gamma_{k}=0
$$

thus

$$
\begin{aligned}
J_{a}\left(u, \mathscr{K}_{i}\right)=\sum_{k=0}^{N-1}\left\{\frac{1}{2} x_{k}^{T}(\right. & \left.Q_{k}+\mathscr{X}_{i} \Gamma_{k}^{T} \Gamma_{k}\right) x_{k}+u_{k}^{T}\left(P_{k}+\mathscr{K}_{i} \Pi_{k}^{T} \Gamma_{k}\right) x_{k} \\
& \left.\quad+\frac{1}{2} u_{k}^{T}\left(R_{k}+\mathscr{K}_{i} \Pi_{k}^{T} \Pi_{i}\right) u_{k}+q_{k}^{\prime}\left(x_{k}, u_{k}\right)\right\}+F\left(x_{N}\right)(7.5
\end{aligned}
$$

where $\Gamma_{k} \in R^{n \times p}, \Pi_{k} \in R^{m \times p}$ and $\gamma_{k} \in R^{p}$ are constant matrices and vectors, respectively and

$$
q^{\prime}\left(x_{k}, u_{k}\right) \triangleq q_{k}\left(x_{k}, u_{k}\right)+\gamma_{k}^{T} \gamma_{k}-\frac{1}{2} \mathscr{X}_{i} \gamma_{k}^{T} \Gamma_{k} x_{k}-\frac{1}{2} \mathscr{K}_{i} \gamma_{k}^{T} \Pi_{k} u_{k}
$$

Since $\Gamma_{k}^{T} \Gamma_{k} \geqslant 0$ and $\Pi_{k}^{T} \Pi_{k} \geqslant 0$ for arbitrary $\Gamma_{k}$ and $\Pi_{k}$, it follows that $Q_{k}+$ $\mathscr{K}_{i} \Gamma_{k}^{T} \Gamma_{k} \geqslant 0$ and $R_{k}+\mathscr{K}_{i} \Pi_{k}^{T} \Pi_{k}>0$. Thus instead of $Q_{k}$ and $R_{k}$ which were used for solving the unconstrained problem (7.1), to solve the approximating problem (7.3) one may use $Q_{k}^{\prime} \triangleq Q_{k}+\mathscr{X}_{i} \Gamma_{k}^{T} \Gamma_{k}$ and $R_{k}^{\prime} \triangleq R_{k}+\mathscr{K}_{i} \Pi_{k}^{T} \Pi_{k}$. Consequently, since the difference between $q_{k}^{\prime}\left(x_{k}, u_{k}\right)$ and $q_{k}\left(x_{k}, u_{k}\right)$ is only in the linear terms (with regard to the LQRE algorithm), the structure of the cost functional in (7.3) is as suitable as that of the cost functional in (7.1). Furthermore, if the problem (7.1) is linear-quadratic and constraint (7.2) linear, LQRE solves the approximating problem in one iteration ( $c f$. Remark 4.2).

\section{References}

[1] P. Dyer and S. R. McReynolds, The computation and theory of optimal control (Academic, New York, 1970).

[2] S. B. Gershwin and D. H . Jacobson, "A discrete-time differential dynamics programming algorithm with application to optimal orbit transfer", AIAA J. 8 (1970), 1616-1626.

[3] M. Heidari, V. T. Chow, P. V. Kokotović and D. D. Meredith, "Discrete differential dynamic programming approach to water resources systems optimization", Water Resources Res. 7 (1971), 273-282. 
[4] D. H. Jacobson and D. Q. Mayne, Differential dynamic programming (Elsevier, New York, 1970).

[5] D. G. Luenberger, Optimization by vector space methods (John Wiley and Sons, New York, 1969).

[6] N. B. Nedeljkovic, "New algorithms for unconstrained nonlinear optimal control problems", IEEE Trans. Automat. Control 26 (1981), 868-884.

[7] K. Ohno, "A new approach to differential dynamic programming for discrete-time systems", IEEE Trans. A utomat. Control 23 (1978), 37-47.

[8] Tennessee Valley Authority, "Development of water resources management methods for the TVA reservoir system", Project status June, 1976. Prelim. Rep. (Knoxville, Tennessee, 1976).

[9] K. L. Teo, K. H. Wong and D. J. Clements, "Optimal control computation for linear time lag systems with linear terminal constraints", J. Optim. Theony Appl. (to appear).

[10] K. H. Wong and K. L. Teo, "A conditional gradient method for a class of time-lag optimal control problems", J. Austral. Math. Soc. Ser. B 25 (1984), 518-537. 